\title{
A cocktail of betulinic acid, parthenolide, honokiol and ginsenoside Rh2 in liposome systems for lung cancer treatment
}

\author{
Xin Jin*,1 (iD, Qing Yang ${ }^{1}$, Ning Cai ${ }^{1}$ \& Zhenhai Zhang**,2 \\ ${ }^{1}$ Department of Hospital Pharmacy, Suqian Branch Jiangsu Province Hospital, 120 Suzhilu, Suqian, 223800, PR China \\ ${ }^{2}$ Jiangsu Province Academy of Traditional Chinese Medicine, 100 Shizijie, Nanjing, 210000, PR China \\ *Author for correspondence: Tel.: +86 15951896676; jinxin871211@163.com \\ **Author for correspondence: Tel.: +86 18913823932; david23932@163.com
}

\begin{abstract}
Aim: Lung cancer has a very high incidence rate, and thus, there is an urgent need for novel and effective therapies. Materials \& methods: In this study, we proposed a potential treatment option by combining four natural products in liposome systems. Results: In vitro studies indicated that the combination of betulinic acid, parthenolide, honokiol and ginsenoside Rh2 exhibited a synergistic action. When these four natural products were loaded into liposome systems, we observed an increased effect. The relative action was also observed in vivo. The cisplatin group presented obvious kidney damage, whereas both cocktail therapy and cocktail liposome therapy were safer. Conclusion: Therefore, we propose cocktail liposome systems may provide a more efficient and safer treatment for lung cancer.
\end{abstract}

\section{Graphical abstract:}

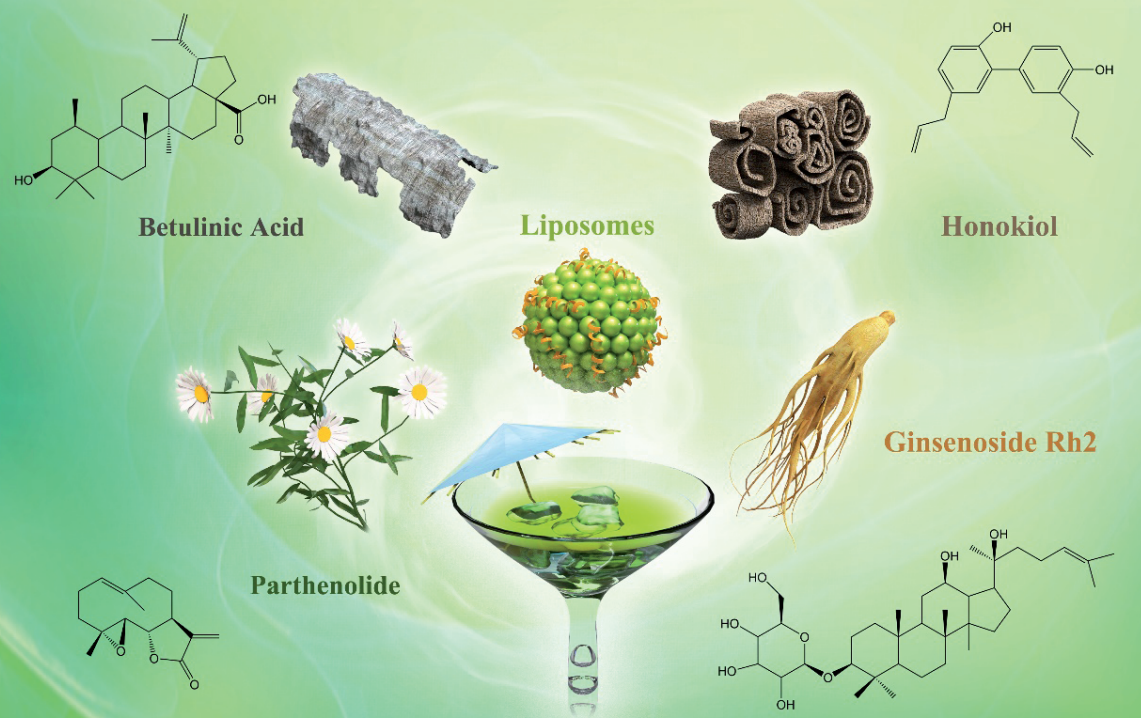

Cocktail Liposomes System

First draft submitted: 12 December 2019; Accepted for publication: 16 October 2019; Published online: 23 December 2019

Keywords: betulinic acid • cocktail therapy $\bullet$ ginsenoside Rh2 • honokiol • liposomes $\bullet$ lung cancer $\bullet$ natural products $\bullet$ parthenolide

Future Medicine 
Chemotherapeutics are commonly used to treat various types of cancer [1]. However, monotherapy often causes drug resistance and poor response in cancer patients after several cycles of treatment. Moreover, it often causes a series of side effects such as bone marrow arrest, gastrointestinal reactions and alopecia, which may decrease patient compliance $[2,3]$. Most traditional Chinese medicines are used as adjuvant therapy for cancer treatment as they present fewer side effects and appropriate antineoplastic activity [4-7]. Complex traditional Chinese medicines that are usually composed of several medicinal herbs can serve as typical representatives of compound medicines. Moreover, they may present better efficacy and decrease unwanted side effects, thereby improving patient survival and compliance [8].

Betulinic acid is a pentacyclic triterpene found in plant sources such as white birch bark [9]. It is known for its ability to directly trigger mitochondrial membrane permeabilization, decrease ATP supply and reduce the efflux of antitumor drugs, which may decrease the resistance to conventional chemotherapeutics [10]. Parthenolide is a lactone, originally purified from the shoots of feverfew (Tanacetum parthenium) [11]. It presents selectivity for cancer stem cells and kills cancer from its roots [12]. Honokiol is a bioactive polyphenol extracted from the roots of Magnolia officinalis [13]. Evidence suggests that this polyphenol can induce autophagy and suppress cancer cell migration [14]. Ginsenoside Rh2 is a dammarane-type saponin obtained from Ginseng [15]. Its antitumor effect is due to its capacity to inhibit the EGFR signaling pathway [16].

However, there are critical limitations to these drugs, such as a lack of specificity, toxic side effects and low solubility. Most of the treatment agents currently administered by therapeutic protocols are systemically circulated without specific localization to cancer tissue. This widespread biodistribution of drugs results in both anticancer activities and off-target adverse activities. Moreover, their poor solubility often leads to difficulty in intravenous administration. Interestingly, it has been suggested that liposome-based codelivery systems, which can increase the solubility of loaded drugs, deliver them to the tumor cells and exhibit synergistic anticancer effects, could be used to overcome the resistance of cancer cells $[17,18]$. As the codelivery systems can simultaneously block two or more pathways, this might promote the death of cancer cells by sensitizing cells to death stimuli [19,20].

Therefore, in this study, a cocktail liposome including these four representative traditional Chinese medicine ingredients (Figure 1A) was chosen and its potential antilung cancer effect was evaluated. Traditionally, the antiviral efficacy of classic cocktail therapy (CT) may be limited by the distinct pharmacokinetic parameters and inconsistent biodistribution of its constituents [21]. Therefore, a well-organized drug delivery system, which can distribute all the constituents simultaneously and effectively, is highly desired [22,23]. Consequently, the development of cocktaildelivery liposomes may be useful. We believe that this strategy for lung cancer treatment holds promise to overcome the limitations and drawbacks of the conventional methods at both research and clinical levels.

\section{Materials \& methods}

Materials

Phosphatidylcholine, 1,2-distearoyl-sn-glycero-3-phosphoethanolamine poly(ethylene glycol) 2000 (DSPE-PEG 2000) and cholesterol were purchased from Shanghai Advanced Vehicle Technology Pharmaceutical Co., Ltd (Shanghai, China). Betulinic acid, parthenolide, honokiol and ginsenoside Rh2 were obtained from Xi'an XiaoCao Botanical Development Co., Ltd (Xi'an, China). Cisplatin was purchased from Nanjing Pharmaceutical Factory Co., Ltd (Nanjing, China). 4',6-Diamidino-2-phenylindole (Hoechst33258) and coumarin-6 were purchased from Shanghai Aladdin Bio-Chem Technology Co., Ltd (Shanghai, China). A549 cells, RPMI 1640 culture medium, fetal bovine serum and fluorescent dyes were obtained from Jiangsu Keygen Biotech Corp., Ltd (Nanjing, China). All the solvents used were of chromatographic grade.

\section{Preparation \& characterization of the cocktail liposome system}

The cocktail liposomes composed of phosphatidylcholine:cholesterol:DSPE-PEG2000 (5:1:4, weight/weight) were prepared by direct hydration of a lipid film as described previously [24]. Briefly, all lipids and drugs (phosphatidylcholine:cholesterol:DSPE-PEG2000:ginsenoside Rh2:parthenolide:betulinic acid:honokiol $=15: 1: 4: 1: 1: 1: 1$, weight/weight) were dissolved with ethanol in a round-bottomed flask, and the solvent was subsequently evaporated using a rotary evaporator under vacuum to afford a dry film. The lipid film was then hydrated using water for $30 \mathrm{~min}$. Next, the lipid was dispersed using probe ultrasound for three cycles to obtain good size distribution. C-6 liposomes were prepared following the same protocol; however, the drugs were partially substituted by coumarin- 6 . 


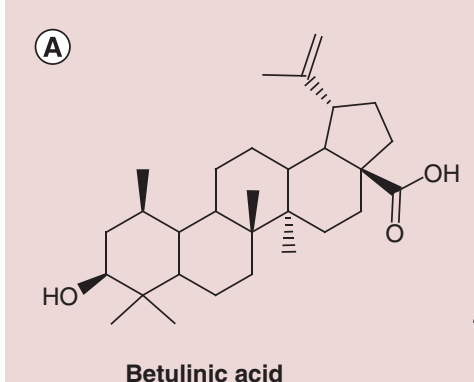

(B)

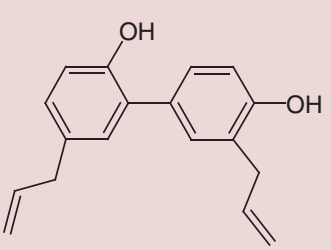

Honokiol

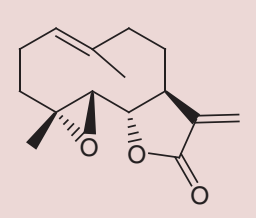

Parthenolide

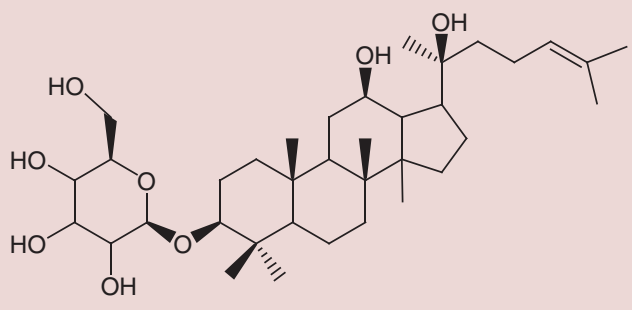

Ginsenoside Rh2

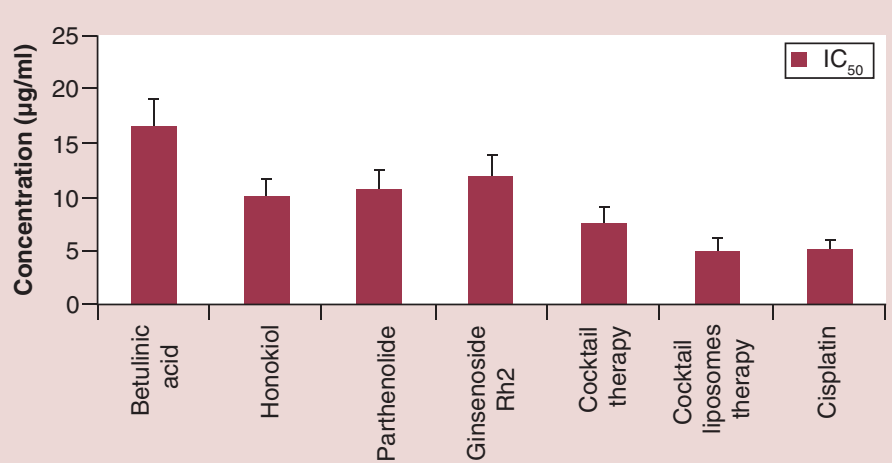

(C)

(D)

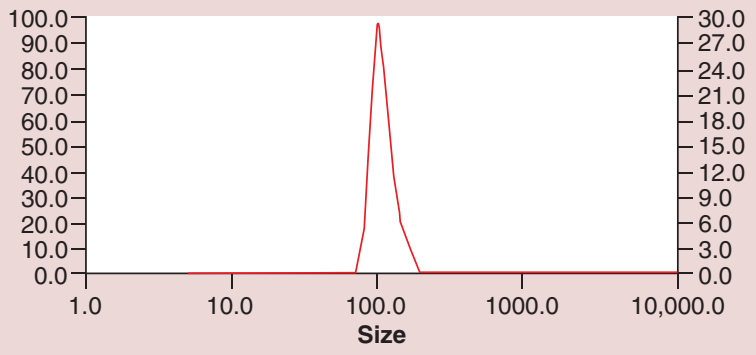

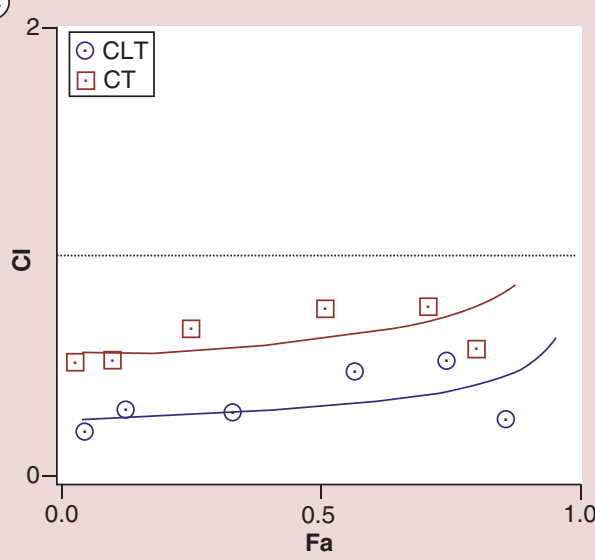

(E)

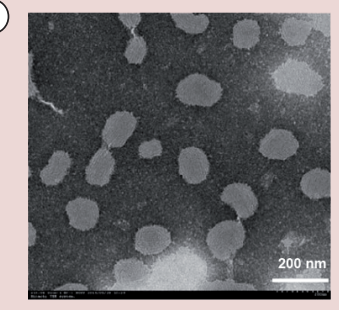

Figure 1. Synergistic effect of cocktail liposomes therapyand its characterization. (A) Chemical structures of betulinic acid, parthenolide, honokiol and ginsenoside Rh2. (B) IC 50 of four natural products, cisplatin, CT and CLT. (C) Cl of CT and CLT. (D) The hydrodynamic size distribution and (E) morphology of cocktail liposomes system was observed by transmission electron microscopy.

$\mathrm{Cl}$ : Combination index; CLT: Cocktail liposomes therapy; CT: Cocktail therapy; Fa: Fraction affected.

\section{In vitro cytotoxicity assay \& its median-effect analysis}

In vitro cytotoxicity against A549 cells was investigated using thiazolyl blue tetrazolium bromide (MTT) cell viability assays. After incubating A549 cells in 96-well plates for $24 \mathrm{~h}$, the culture medium was replaced with betulinic acid, parthenolide, honokiol, ginsenoside $\mathrm{Rh} 2$, cisplatin, CT (four natural products at the weight ratio of 1:1:1:1), or cocktail liposome therapy (CLT; four natural products at the weight ratio of 1:1:1:1). MTT assays were carried out after incubation for $72 \mathrm{~h}$. The effects of drug combinations were analyzed using the CalcuSyn software (Biosoft, Cambridge, UK). The combination index (CI) was calculated by using the Chou-Talalay method for the evaluation of the synergism $(\mathrm{CI}<1)$ or antagonism $(\mathrm{CI}>1)$ of each drug combination [25].

\section{Average hydrodynamic size \& morphological analysis}

The average size and $\zeta$-potential were measured by dynamic light scattering (Mastersizer 2000, Malvern, UK) at a sample temperature of $25^{\circ} \mathrm{C}$ after equilibration for $5 \mathrm{~min}$. All liposome dispersions were diluted with distilled water (1:10) prior to the measurements.

The liposome system was examined for its morphological characteristics using transmission electron microscopy (JEM-100 CX, JEOL, Tokyo, Japan). A 100-fold diluted dispersion of the liposomes in distilled water was mounted 
on a carbon grid. The dispersion was stained with uranyl acetate solution for $1 \mathrm{~min}$. Excess stain was removed using a filter paper, and the sample was left to air dry.

\section{Encapsulation efficiency \& total loading efficiency}

The drug loading (DL) and encapsulation efficiency (EE) of the drug-loaded liposomes were evaluated by a centrifugal method. Briefly, a predefined amount of cocktail liposome was centrifuged at $3000 \mathrm{rpm}$ for 15 min to remove free drugs. The supernatant was collected, dispersed in acetonitrile, centrifugated at $3000 \mathrm{rpm}$ for $15 \mathrm{~min}$ and filtered through a $0.45-\mu \mathrm{m}$ membrane filter to determine the drug content by HPLC.

The EE\% and DL\% values were calculated as follows:

$\mathrm{EE}(\%)=$ mass of a drug in the liposomes $/$ mass of this drug loaded initially $\times 100$

$\mathrm{DL}(\%)=$ mass of all drugs in the liposomes $/$ mass of cocktail liposome $\times 100$

\section{Cellular uptake}

A549 cells were seeded in a 12-well plate and cultured for $24 \mathrm{~h}$. Next, coumarin-6 or coumarin-6-labeled liposomes were added and cultured for 4 and $8 \mathrm{~h}$ at the same concentration. The cells were washed three times with blank phosphate-buffered saline (PBS) and then stained for $20 \mathrm{~min}$ at $37^{\circ} \mathrm{C}$ using LysoTracker Red $(25 \mathrm{ng} / \mathrm{ml})$ to detect endosomes/lysosomes and Hoechst $33258(10 \mu \mathrm{g} / \mathrm{ml})$ to detect nuclei. The cells were then washed three-times with PBS. The cellular localization was visualized by confocal laser scanning microscopy (Zeiss, Jena, Germany).

5-ethynyl-2'-deoxyuridine-staining assay

A549 cells $\left(2 \times 10^{4}\right)$ were seeded in 24-well culture plates and treated with betulinic acid $(6 \mu \mathrm{g} / \mathrm{ml})$, parthenolide $(6 \mu \mathrm{g} / \mathrm{ml})$, honokiol $(6 \mu \mathrm{g} / \mathrm{ml})$, ginsenoside Rh2 $(6 \mu \mathrm{g} / \mathrm{ml})$, cisplatin $(6 \mu \mathrm{g} / \mathrm{ml})$, CT $(6 \mu \mathrm{g} / \mathrm{ml}$ total four natural products at the weight ratio of $1: 1: 1: 1)$ or CLT $(6 \mu \mathrm{g} / \mathrm{ml}$ total four natural products at the weight ratio of $1: 1: 1: 1)$ for $72 \mathrm{~h}$. Then, 5-ethynyl-2'-deoxyuridine (EdU) was added to the medium (final concentration: $50 \mu \mathrm{M}$ ) for $2 \mathrm{~h}$. The cells were then washed twice with PBS and fixed with anhydrous ethanol for $30 \mathrm{~min}$. Subsequently, the cells were incubated with Apollo ${ }^{\circledR}$ for 30 min. Finally, the cells were stained by Hoechst 33342 for 15 min. A high intension cell imaging system was used to obtain fluorescent images.

\section{Flow cytometric analysis}

The effects of CLT on cell cycle and apoptosis were detected via flow cytometric analysis as previously reported [24]. Briefly, after treatment with the different formulations for $72 \mathrm{~h}$, the cells were trypsinized and the cell pellet was obtained; the supernatant was washed with ice-cold PBS. The cells were stained with propidium iodide and a 3',6'-dihydroxy-5-isothiocyanatospiro(isobenzofuran-1 [3H],9'-[9H]xanthen)-3-one (FITC)-Annexin V Apoptosis Detection kit was used. Finally, the binding buffer was analyzed by flow cytometry.

Imaging of intracellular $\mathrm{Ca}^{2+}$

After treatment with the different formulations for $72 \mathrm{~h}, \mathrm{~A} 549$ cells were dyed with Fluo-3 AM $(2.5 \mathrm{Y} \mathrm{mM})$ at $37^{\circ} \mathrm{C}$ for $1 \mathrm{~h}$. Fluorescence images were obtained at an excitation wavelength (Ex) of $488 \mathrm{~nm}$ and emission wavelength (Em) of $506 \mathrm{~nm}$.

\section{Wound-healing \& cell invasion assay}

A549 cells were seeded in a 24-well plate and cultured for $24 \mathrm{~h}$. Then, the cell monolayers were scratched with a $20-\mu \mathrm{l}$ pipet tip before treatment with betulinic acid, parthenolide, honokiol, ginsenoside Rh2, cisplatin, CT or CLT. After incubation for $24 \mathrm{~h}$, wound healing was recorded. Images were obtained using a microscope both before and after $24 \mathrm{~h}$ to further evaluate cell migration.

To assess the capacity for invasion, the A549 cells were seeded on the upper chambers that were precoated with Matrigel in the Transwell inserts. RPMI-1640 medium containing 10\% fetal bovine serum was added to the lower chamber containing the different therapeutic agents for $24 \mathrm{~h}$. Subsequently, the cells that invaded the bottom of the membrane were stained with crystal violet solution for $30 \mathrm{~min}$ and washed twice with PBS. The stained cells were visualized under a microscope (Olympus, Tokyo, Japan).

\section{In vivo antitumor efficacy}

A549 lung cancer cells were injected subcutaneously into the flank region (right front) of nude mice. Tumor bearing mice $\left(\sim 50 \mathrm{~mm}^{3}\right)$ were randomized into four groups (six mice/group); saline as control, cisplatin $(10 \mathrm{mg} / \mathrm{kg})$ as 
positive control, CLT (ginsenoside Rh2, parthenolide, betulinic acid and honokiol at 20, 20.5, 20.3 and $20.7 \mathrm{mg} / \mathrm{kg}$, respectively) and CT (in doses identical to the doses for CLT) were administered intravenously every 3 days for nine cycles. Tumor volumes and bodyweights were examined every 3 days. Tumor samples were obtained using Vernier calipers and the volumes were calculated. From each group, all mice were sacrificed by cervical vertebra dislocation on day 28 to harvest main tissues for further histopathological examination. The tissues were stored in $4 \%$ paraformaldehyde for 1 week, embedded in paraffin and cut into sections. The paraffin-embedded sections were used to determine Ki67 levels by using immunohistochemical staining, hematoxylin and eosin (H\&E) staining and terminal deoxynucleotidyl transferase dUTP nick-end labeling (TUNEL) assay.

\section{Statistical analysis}

The data are presented as means \pm standard error of mean. Differences among groups were determined using one-way analysis of variance (ANOVA) followed by Tukey's post-test. A p-value less than 0.05 indicated statistical significance.

\section{Results}

\section{Effect against $A 549$ cells}

The $\mathrm{IC}_{50}$ values of the different natural products and the codelivery system were evaluated using A549 cells, as shown in Figure 1B, before testing the CI. All the natural products evaluated in this study inhibited tumor growth. However, the antitumor effect was smaller than that observed with cisplatin. The use of all the drugs together in the CT further increased tumor growth inhibition; however, the effect was still smaller than that observed with cisplatin. Interestingly, with liposome codelivery, the antitumor effect was increased, presenting results comparable to the ones observed in the cisplatin group. To further study the synergistic effect of the CT, the dose-response curves of different natural products and the codelivery system are shown in Supplementary Figure 1. The CI was quantitatively analyzed using the CalcuSyn software (as shown in Figure 1C). Although both CT $(C I=0.65)$ and CLT $(\mathrm{CI}=0.37)$ presented a synergistic effect $(\mathrm{CI}<1)$, the synergistic effect of the CLT was higher than that of the CT, indicating an increased potential and feasibility of this combination.

\section{Preparation \& characterization of the cocktail liposome system}

The average hydrodynamic size of the cocktail liposome system was $115.7 \mathrm{~nm}$ (as shown in Figure 1D) with a $\zeta$-potential of - $18.31 \mathrm{mV}$. The EE was 88.2, 90.3, 89.5 and $91.4 \%$ for ginsenoside Rh2, parthenolide, betulinic acid and honokiol, respectively, and the total loading efficiency was $15.3 \%$. The morphology of the cocktail liposome system was consistent with the typical spherical morphology of liposomes (as shown in Figure 1E). The designed liposome system, when stored at room temperature for 1 week, showed no significant change in the sizes, inferring stability to storage.

\section{Cellular uptake}

As shown in Figure 2, cellular uptake was time-dependent and the liposome system presented a higher cellular uptake than free coumarin- 6 due to cell endocytosis.

The coumarin- 6 located inside the lysosomes was yellow because of an overlap between the green (coumarin-6) and red (LysoTracker) fluorescent spots. Figure 2 also shows that coumarin-6 induced green fluorescence and colocalization were identical to the lysosome group both at 4 and $8 \mathrm{~h}$, indicating an uptake into the lysosomes upon cell internalization. However, although it exhibited colocalization within lysosomes in $4 \mathrm{~h}$, the colocalization decreased significantly after $8 \mathrm{~h}$, suggesting that the liposome system could escape lysosomes. This may be because the liposome system assembled from lipids can fuse with the endosomal membrane, inverting the structure of the liposome and delivering the therapeutic core to the cytoplasm [26]. Furthermore, the quantitative analysis for cellular uptake (Supplementary Figure 2) was consistent with that of the observed images. Therefore, this effect could be the reason for an improvement in the absorption of the drugs.

\section{EdU staining}

The EdU staining assay was performed to investigate cell proliferation. Although all four natural products could decrease the percentages of EdU-positive cells (pink), indicating inhibition of cell proliferation, the cisplatin and CLT groups presented a higher inhibitory effect. Both CT and CLT exhibited an antilung cancer action (as shown in Figure 3A \& Supplementary Figure 3). The percentages of EdU-positive cells treated with cisplatin and with CLT were 22.34 and $28.67 \%$, respectively $(\mathrm{p}<0.05)$. 


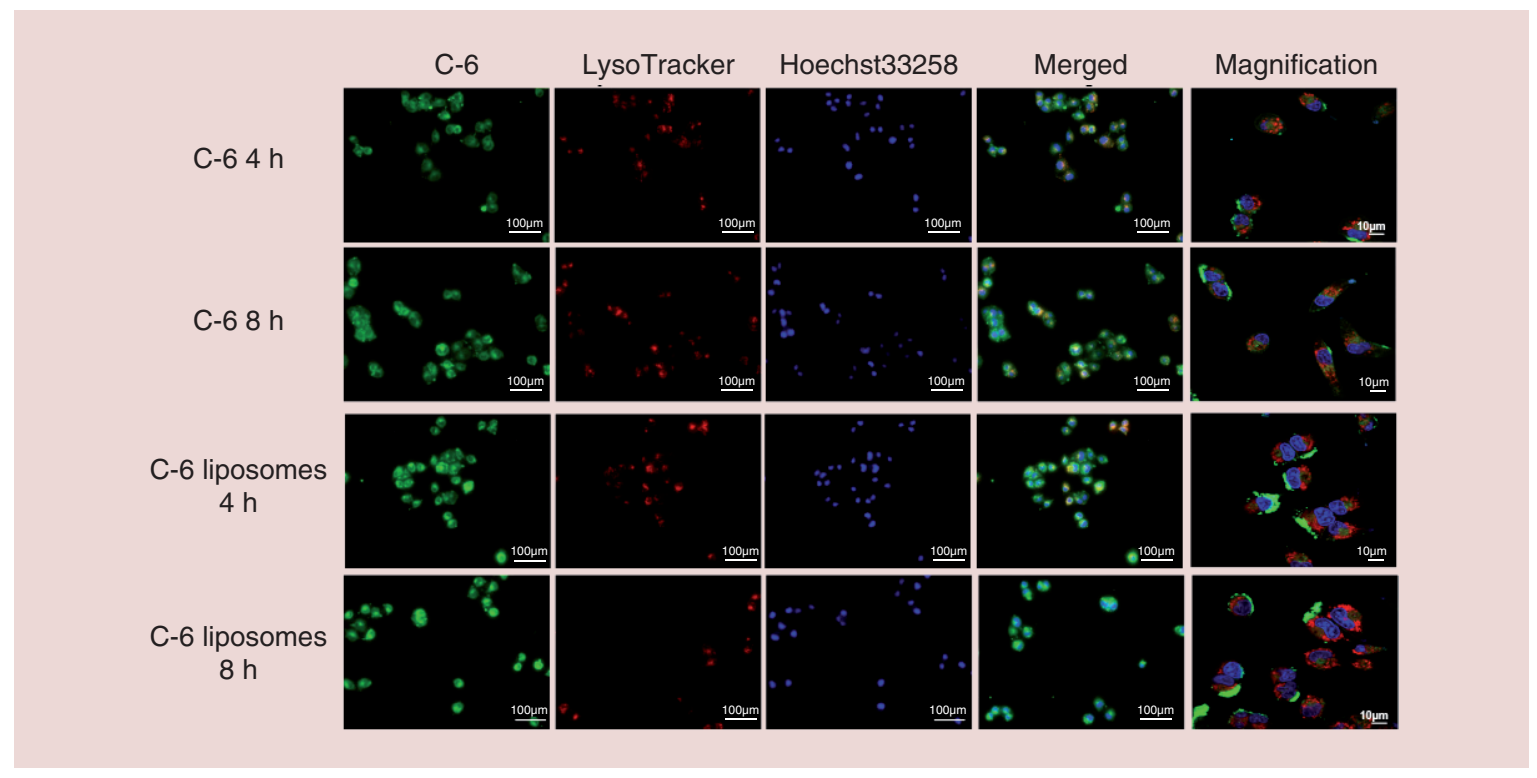

Figure 2. Uptake and lysosomes escape of liposomes system at 4 and $8 \mathrm{~h}$.

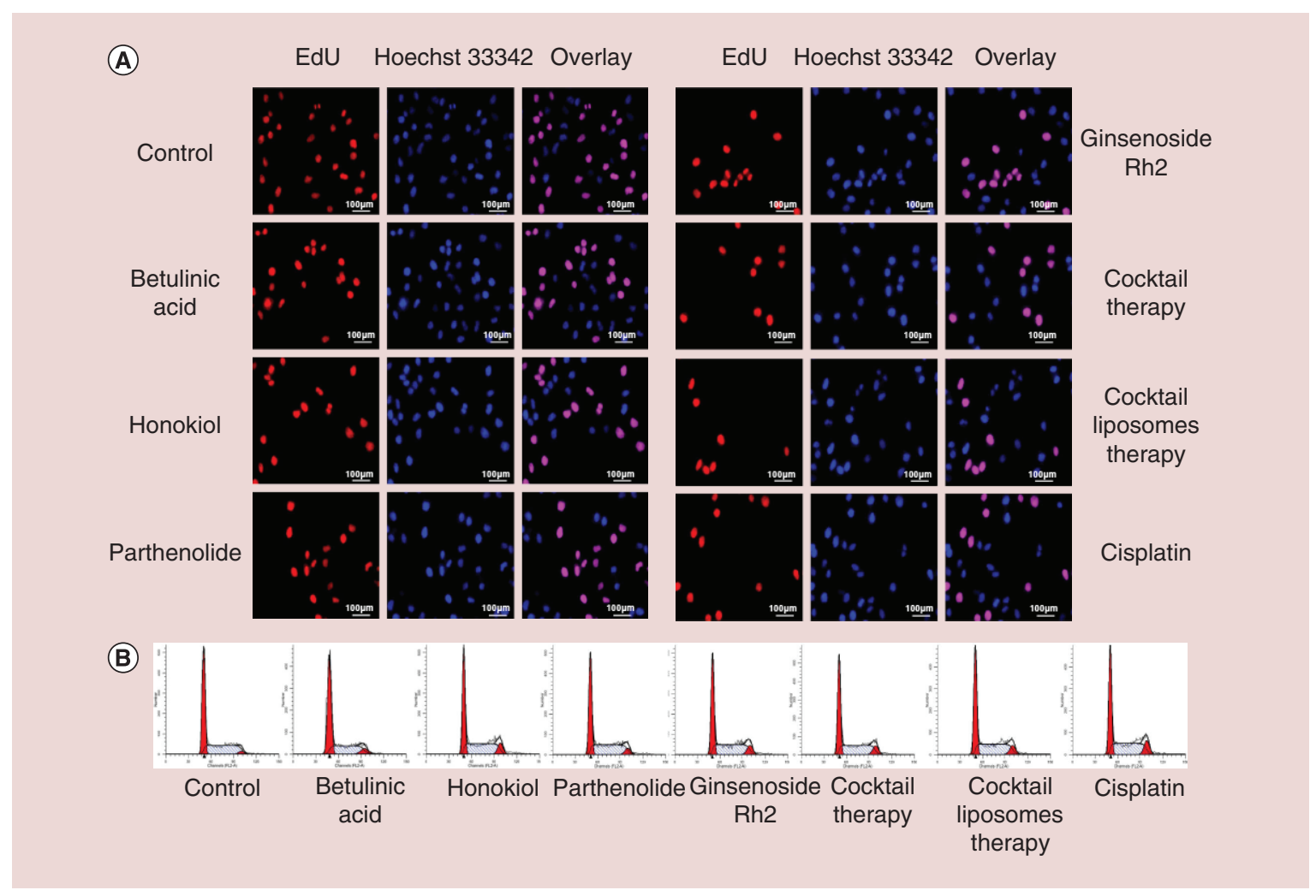

Figure 3. Proliferation inhibition and cell cycle arrested. EdU staining (A) and cell cycle arrested (B) of cocktail liposome therapy. 
(A)
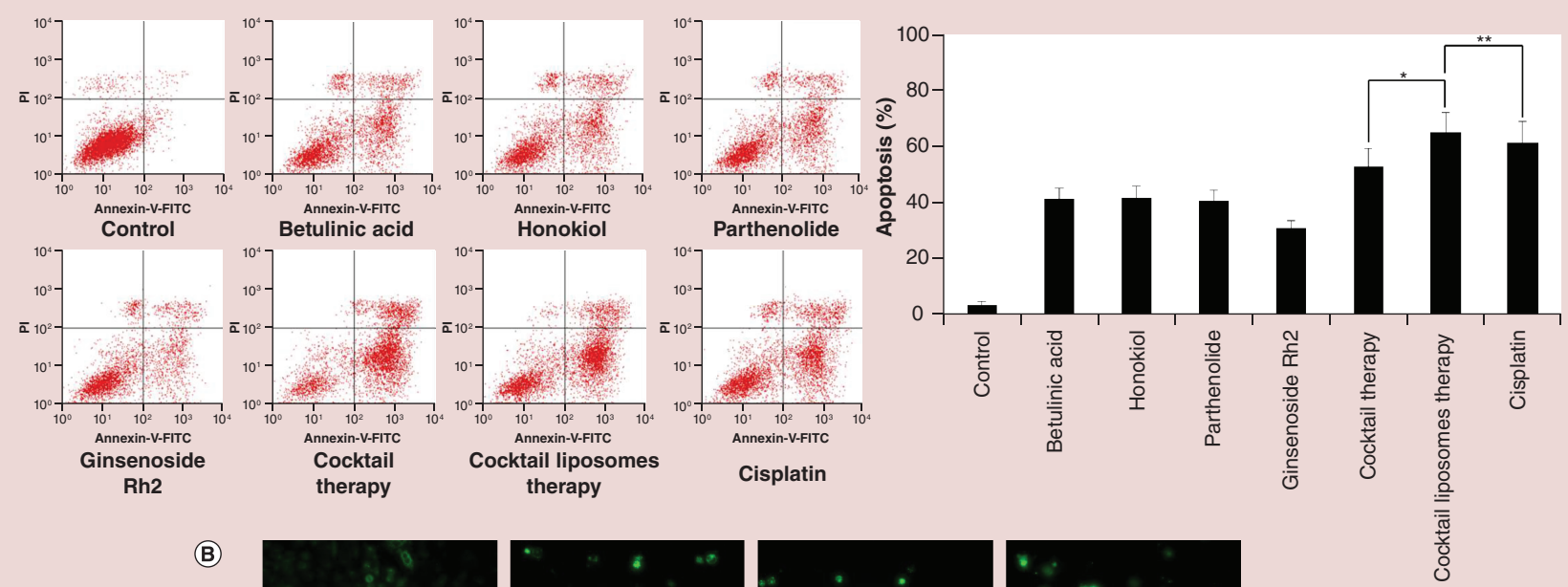

(B)
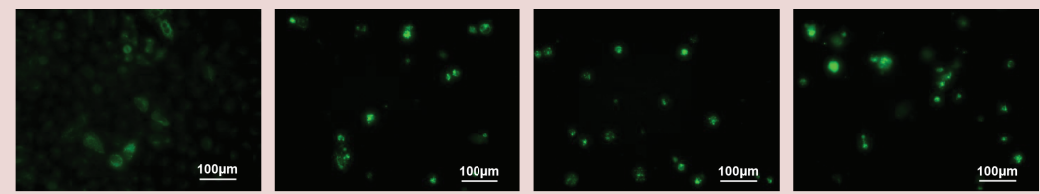

Control

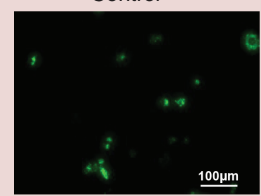

Betulinic acid

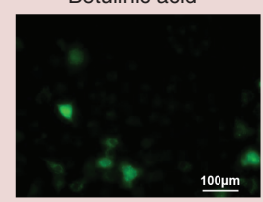

Honokiol

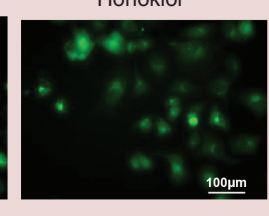

Parthenolide

Ginsenoside

Rh2

Cocktail therapy

Cocktail liposomes

therapy

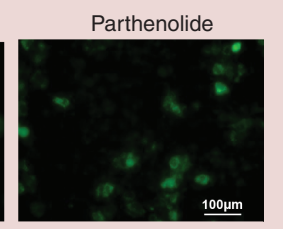

Cisplatin

Figure 4. Induced cell apoptosis and $\mathrm{Ca}^{2+}$ distribution. (A) Induced cell apoptosis; ${ }^{*} \mathrm{p}<0.05$ cocktail therapy versus the cocktail liposomes therapy, ${ }^{* *} \mathrm{p}<0.05$ cisplatin versus the cocktail liposomes therapy. (B) Intracellular $\mathrm{Ca}^{2+}$ distribution of cocktail liposome therapy.

\section{Cell cycle arrest \& induction of apoptosis}

The experimental drugs and cisplatin could arrest the cells in the G2/M phase (as shown in Figure 3B). The results also revealed that cocktail liposome could efficiently block the cell cycle in the G2/M phase, similar to that observed after cisplatin treatment.

The growth of cancer cells is affected by two major factors: proliferation and apoptosis. The aforementioned results indicate that CLT could suppress A549 cell proliferation efficiently. However, its potential role in apoptosis of A5 49 cells was unclear. Therefore, the apoptosis ratios of A549 cells were further evaluated (as shown in Figure 4A). The apoptosis rates were 31.1, 35.6, 30.4 and $21.6 \%$ following treatment with betulinic acid, parthenolide, honokiol and ginsenoside Rh2, respectively. Cisplatin, CT and CLT induced apoptosis to a greater extent than the free individual drugs $(61.1,55.7$ and $63.6 \%$, respectively).

\section{Mitochondrial function of cancer apoptosis}

To further understand cancer cell apoptosis induced by these therapies, the mitochondrial pathway was analyzed by determining intracellular distribution of $\mathrm{Ca}^{2+}$ (as shown in Figure 4B). Increased $\mathrm{Ca}^{2+}$ levels (green clusters) revealed that the cancer cells underwent mitochondrial apoptosis. In the control cells, mild green fluorescence indicated no significant apoptosis was induced by the mitochondria in this group. Meanwhile, combination treatment with the four natural products increased the level of green fluorescence, indicating increased mitochondrial apoptosis. Moreover, following CLT, the green fluorescence was stronger than that after cisplatin therapy, indicating that cisplatin may induce cancer cells apoptosis via multiple pathways apart from mitochondrial apoptosis. Furthermore, the quantitative analysis of intracellular $\mathrm{Ca}^{2+}$ distribution (Supplementary Figure 4) was consistent with that of the observed images. 


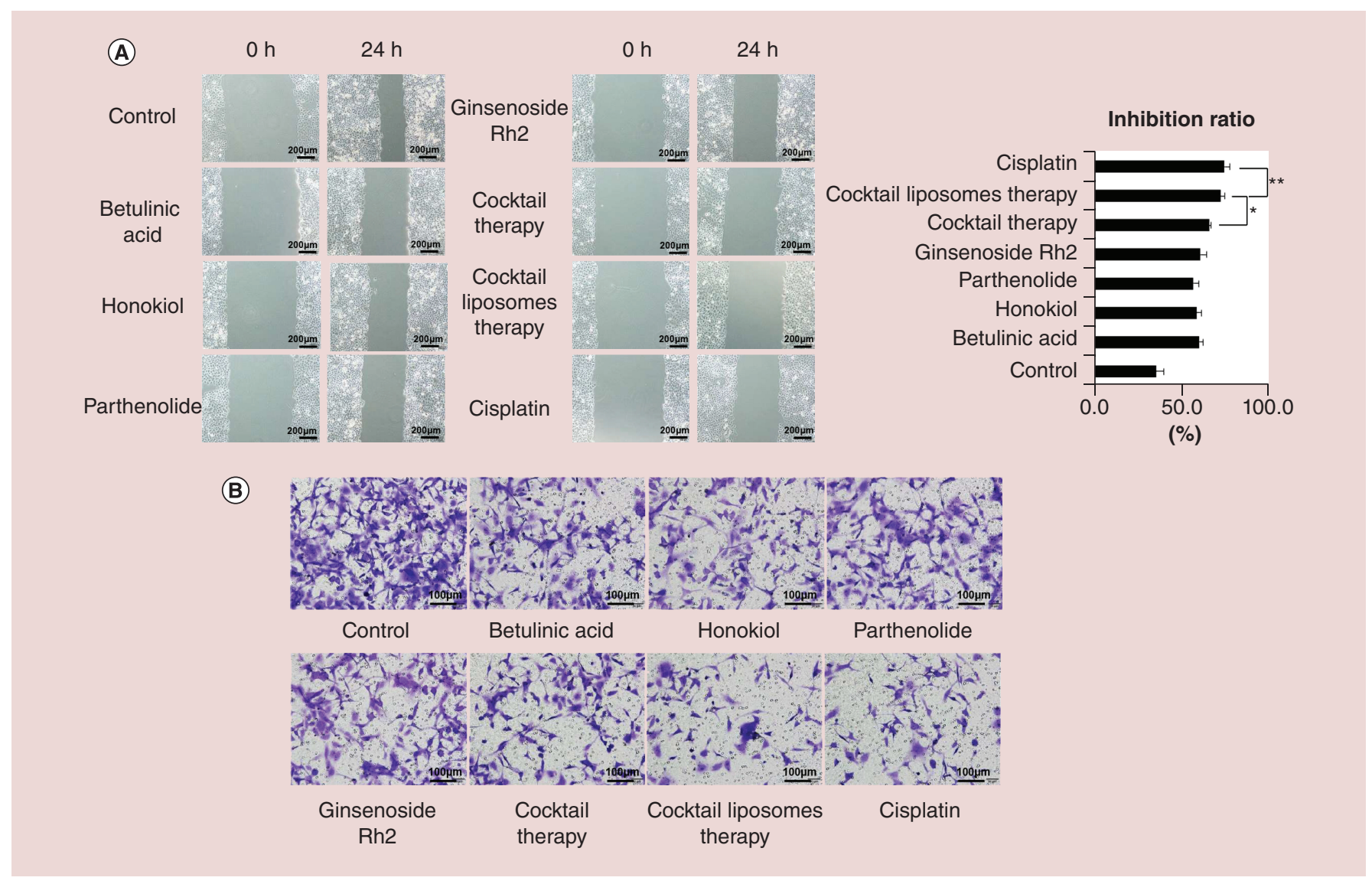

Figure 5. Inhibition metastasis. (A) Wound-healing inhibition; ${ }^{*} p<0.05$ cocktail therapy versus the cocktail liposome therapy, ${ }^{*} p$ $<0.05$ cisplatin versus the cocktail liposome therapy. (B) Transwell migration decrease.

Wound healing \& transwell migration

The CLT strongly prevented A549 cell migration, similar to cisplatin; the effect was remarkably higher than that observed in the other groups (as shown in Figure 5A). Cisplatin and CLT inhibited cell migration by 74.7 and $72.6 \%$, respectively. The free natural products revealed inhibition rates less than $60 \%$. Consistently, similar results were found in the transwell assay. There was a significant decrease in cancer cells passing through the transwell membrane filter when treated with the cocktail liposomes. Codelivery of the four natural products via liposome showed the best effect.

\section{Xenografted tumor growth in vivo}

The antitumor efficacy of the CT and CLT was further evaluated in vivo. Cisplatin was chosen as the positive control. After treatment, tumors were obtained and evaluated (Figure 6A). The tumor inhibition rates of cisplatin, CT and CLT were 65.1, 42.5 and 60.7\%, respectively. Treatment with cisplatin or the two cocktail therapies could significantly decrease tumor growth compared with control treatment $(\mathrm{p}<0.05)$. Meanwhile, treatment with CLT could significantly decrease tumor growth compared with CT ( $\mathrm{p}<0.05)$.

Although cisplatin showed the best tumor inhibitory effect, the tumor inhibition rate of the cocktail liposome group was only $4.40 \%$ smaller than that of the cisplatin group (as shown in Figure 6B). No significant difference in bodyweight (Figure 6C) was observed among groups.

To further investigate the effects of the CLT, proliferation, necrosis and apoptosis levels in tumor sections were analyzed by determining Ki67 levels using immunohistochemistry, H\&E staining and TUNEL assay (Figure 6D). Rate of cell proliferation in the tumor sections measured by the Ki67 assay was lower after treatment with cocktail liposomes $(3.41 \pm 0.16 \%)$ than after treatment with cocktail $(7.52 \pm 0.83 \%)$ and with control $(13.26 \pm 1.67 \%)$; proliferation rates were comparable between the cocktail liposomes and the cisplatin $(2.68 \pm 0.29 \%)$ groups. 
(A)

(B)
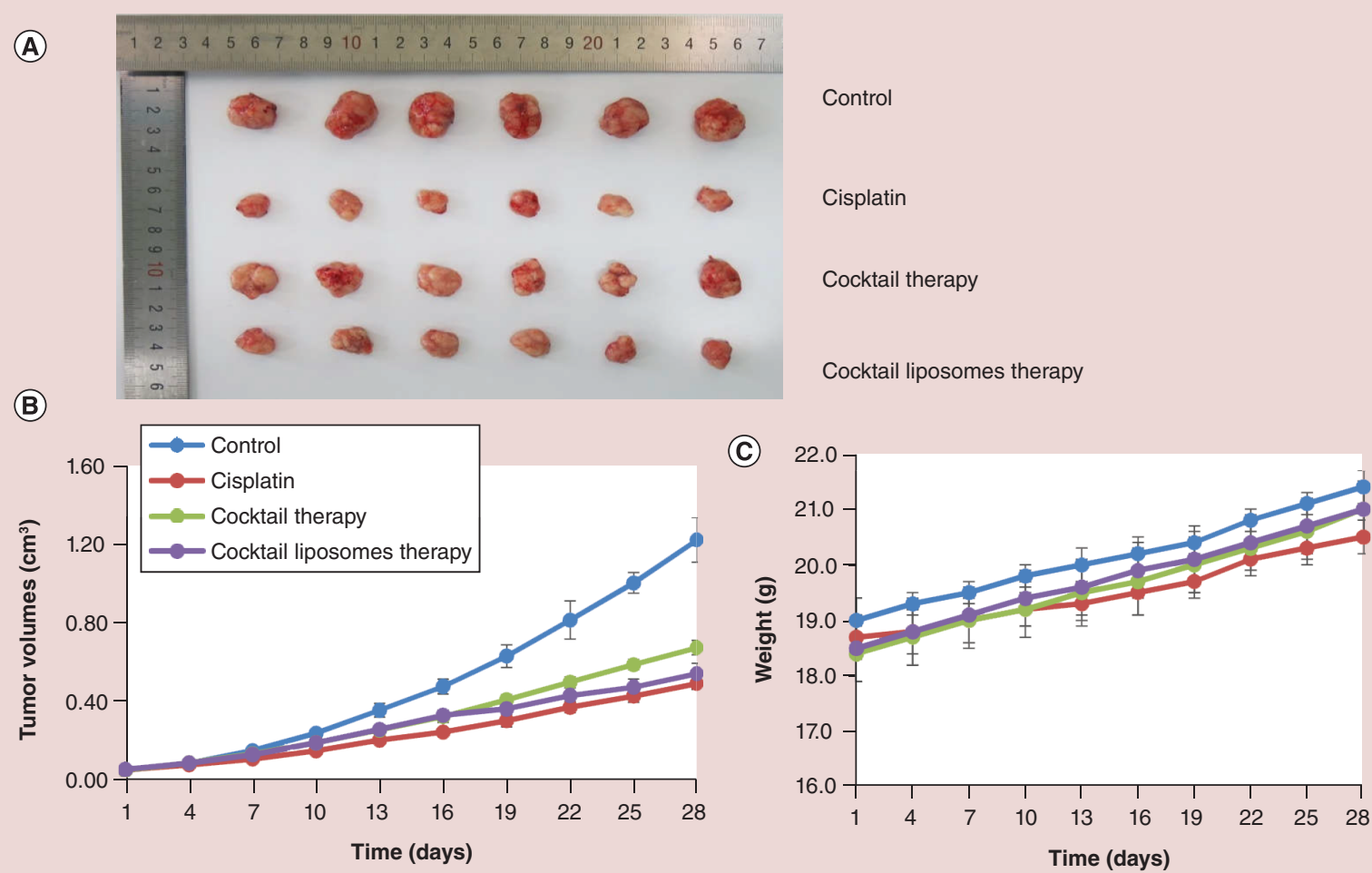

(D)

Ki67

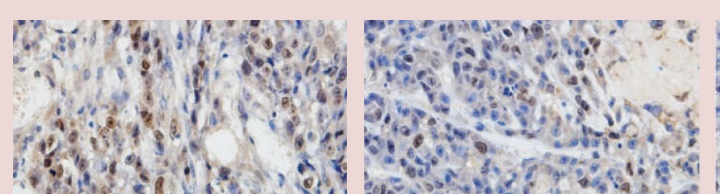

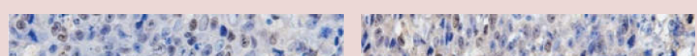

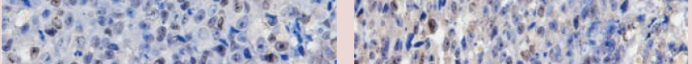
5.

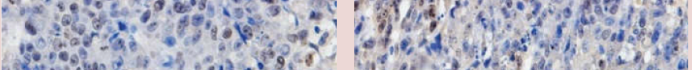

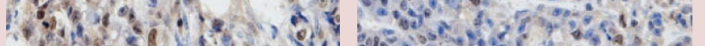
a If

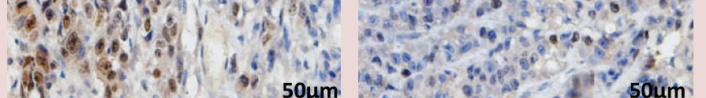
Gog

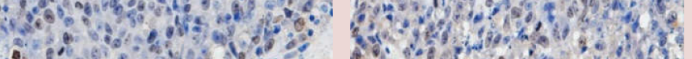
60 a

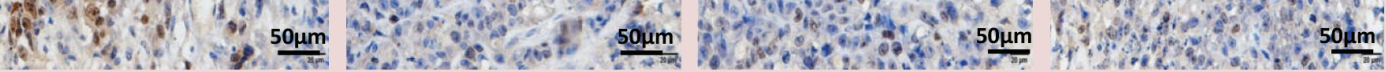

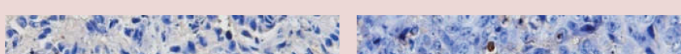

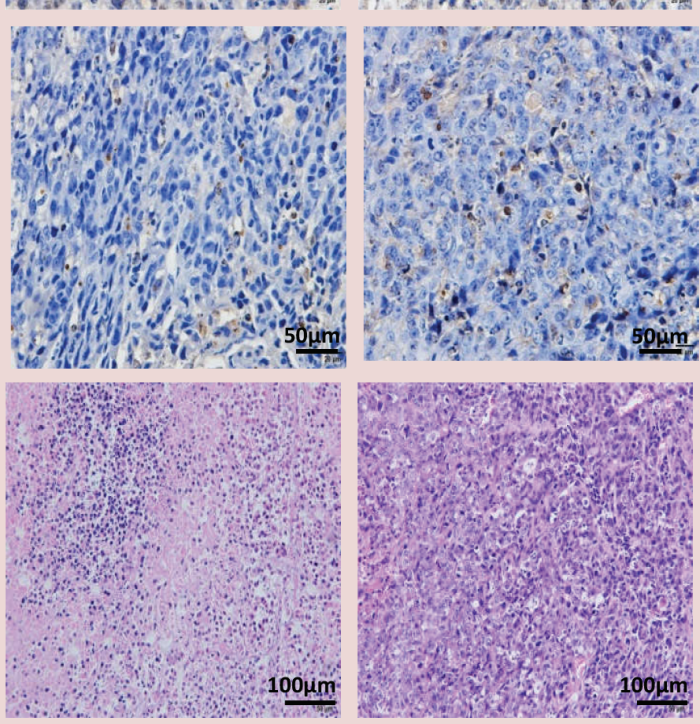

TUNEL
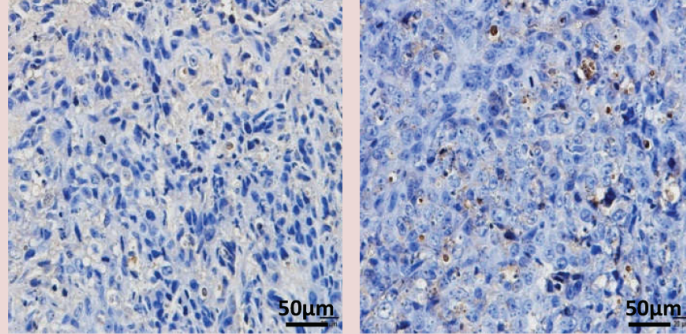

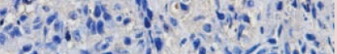
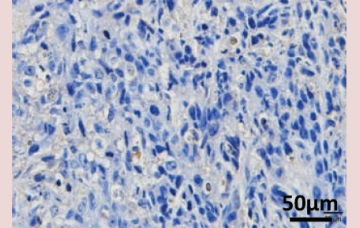

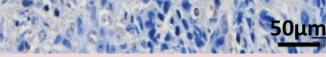
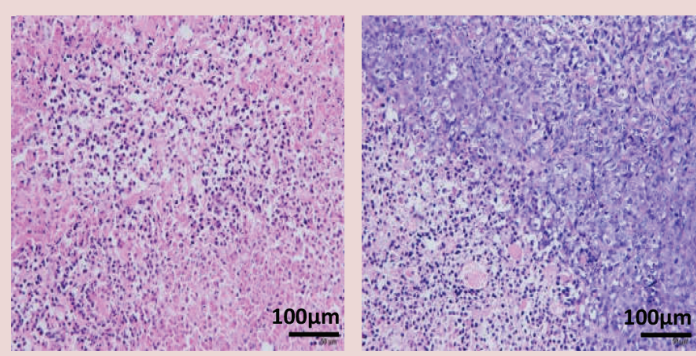

Control

Cisplatin

$$
\begin{aligned}
& \text { Cocktail } \\
& \text { therapy }
\end{aligned}
$$

Cocktail liposomes therapy

Figure 6. In vivo antitumor effect. (A) At the end of the study, tumors were photographed and (B) the volumes were recorded. (C) Average bodyweight of the mice was obtained every 3 days. (D) Immunohistochemistry of Ki67, H\&E stain and the terminal deoxynucleotidyl transferase dUTP nick end labeling.

H\&E: Hematoxylin and eosin. 


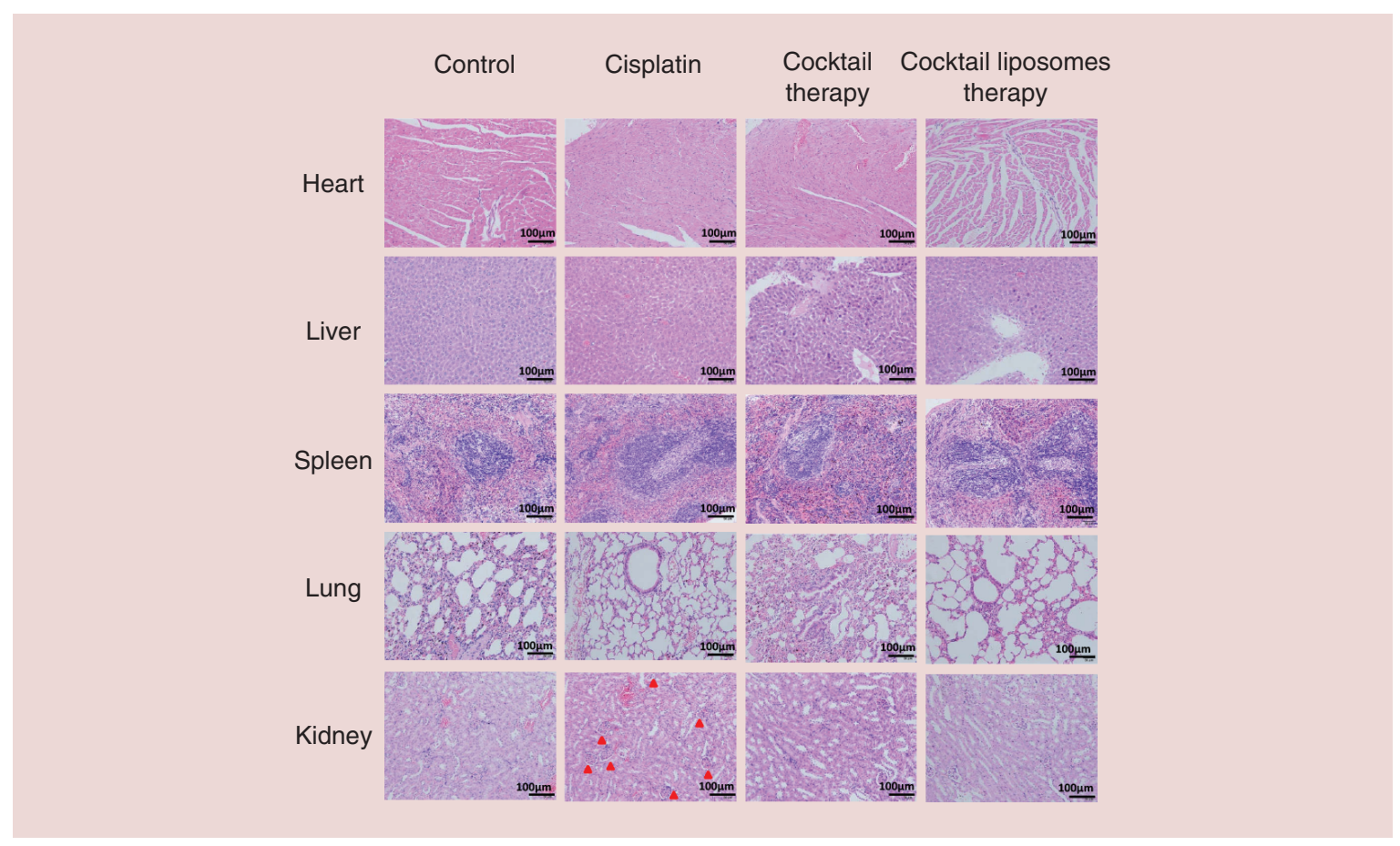

Figure 7. Hematoxylin and eosin stain of hearts, livers, spleens, lungs and kidneys of mice at the end of experiments.

Meanwhile, in the control group, a large area of necrotic hollows was observed, which indicated a stronger proliferation effect. After treatment with different formulations, a decrease in necrosis was observed in the CT group; however, this effect was more pronounced in the CLT and cisplatin groups, indicating better inhibition of the cancer cell proliferation in the CLT group. The apoptosis rate was determined using TUNEL assay. The group treated with the cocktail liposomes $(10.15 \pm 1.92 \%)$ presented a higher percentage of apoptosis than the group treated with the cocktail $(5.31 \pm 0.87 \%)$, and an identical percentage to that of the cisplatin group (12.53 and an identical percentage to that of $\pm 2.12 \%$ ). These results suggest that decreased proliferation and increased apoptosis might be the main factors driving the decrease in tumor growth in vivo, consistent with the results obtained in vitro. Despite the better antitumor effects of the CLT, the side effects of this formulation cannot be ignored. To assess its therapeutic index, the tissues were stained with H\&E. As shown in Figure 7, necrosis of the renal tubular epithelial cells was only observed in the cisplatin group. Therefore, we can infer that both CT and CLT did not cause any significant damage to the major organs, whereas cisplatin induced obvious kidney damage, making this cocktail a safer option for lung cancer treatment.

All these findings demonstrate that the CLT may be an efficient alternative for lung cancer treatment with fewer side effects.

\section{Discussion}

Cisplatin is the first-line therapy for lung cancer. However, its renal toxicity, adverse gastrointestinal effects and myelosuppression restrict its application in the long term. A combination of carboplatin, nedaplatin and oxaliplatin developed to curb the adverse effects of cisplatin had similar side effects. Moreover, single-drug treatment is often limited by the maximal tolerated dose, which often leads to inefficient treatment due to the physiological complexity of the tumor and adverse effects. Therefore, the 5-year survival rate of the lung cancer patient is still unsatisfactory.

When drug cocktails are used to treat lung cancers, unlike single drugs that inhibit tumor growth by interfering with a single mechanism or several limited channels, they can modulate several cellular mechanisms to obtain high efficacy. Although the new drugs developed often improve the response rate, duration of response and patient survival, the lack of specific tumor accumulation may restrict their effect. In order to avoid this important issue, the US FDA recommendations have been published supporting the codelivery of a combination of two or more of novel investigational drugs, emphasizing the crucial role of this powerful approach to treat patients. Different 
nanocarriers, such as mixed micelles [27], polymeric nanoparticles [28] and liposomes [29], have been designed to improve antitumor effects. Based on the advantage of liposomes, currently, a new cocktail chemotherapy by combining drugs and liposome systems is being clinically tested and explored to improve therapeutic efficacy and minimize side effects [30-33]. Therefore, the design of natural product liposome systems has its merits.

Natural products represent an excellent library of bioactive molecules. Thus, accessing the natural world may prove useful for the identification of new therapeutic options with favorable profiles [34-36]. Recently, an increasing emphasis has been placed on cancer therapies separately or concurrently with herbs and/or their products [37,38]. Four types of natural products including a pentacyclic triterpene, a lactone, a polyphenol and a dammaranetype saponin were chosen to target different mechanisms. These complex multiple mechanisms may include the suppression of the growth of cancer cells [39], the induction of apoptosis [40,41], the inhibition of cancer stem cells [42], the suppression of tumor metastasis [43], the reversal of resistance [44,45] and the promotion of the efficacy of other anticancer drugs [46]. Then, a liposome system was chosen as a carrier of these active agents. From the in vitro experiments, it was evident that the simple CT could obtain a better antilung tumor effect; however, the CLT could promote increased cellular uptake of the loaded cargo and escape lysosomes, leading to improved effects. Additionally, MTT assay; EdU staining; and cell cycle, apoptosis, mitochondrial function and migration assays confirmed that the CLT had enhanced therapeutic effects by various functions.

Moreover, approximately $100 \mathrm{~nm}$ liposomes can penetrate into tumor cells [47]. Unlike most of the normal tissues, tumor vasculature is heterogeneous and does not match the standard morphologies [48]. The natural products are selectively leaked from the tumor vessels and accumulate in tumor tissues for prolonged periods [49]. Nanoleak in tumor vessels enabled natural products contained in liposomes with the opportunity to more efficiently target the tumor by enhancing their permeability and retention effect. Intravascular administration of PEG-modified liposome systems could avoid recognition by the reticuloendothelial system and increase the efficacy of therapy [50,51].

\section{Limitations}

Although the CT with betulinic acid, parthenolide, honokiol and ginsenoside Rh2 in liposomes could cause an ideal effect, the potential limitations of our study should also be considered. The ratio of the four natural drugs was not optimized. Single drug liposome systems were not designed and used for further evaluation. Further studies regarding these issues are currently underway. Even if these limitations exist, CT with safe natural products in liposomes is still beneficial against lung cancer.

\section{Conclusion}

The development of novel and efficient treatment strategies for lung cancer has vital importance considering its treatment refractoriness and high incidence rate. In summary, this study provides a strategy for the combination of multiple natural products and liposomes instead of traditional chemotherapy for lung cancer. To our knowledge, this approach is novel and by tailoring the synergistic effects of its constituents, it has improved safety and efficacy.

\section{Summary points}

- Traditional Chinese medicines are used as adjuvant therapy for cancer as they present both fewer side effects and appropriate antineoplastic activity.

- The combination of betulinic acid, parthenolide, honokiol and ginsenoside Rh2, loaded into liposome systems, can be successfully prepared.

- The combination index of cocktail therapy and cocktail liposome therapy was 0.65 and 0.37 , respectively, and indicated a synergistic effect $(\mathrm{Cl}<1)$.

- The cellular uptake of the liposome system was time-dependent and presented lysosome escape behavior.

- Cocktail liposome therapy presented significant cytotoxicity to lung cancer cells in vitro and in vivo with less side effects. 
Financial \& competing interests disclosure

This work was supported by Project of Jiangsu Natural Science Foundation of China (BK20171321), Jiangsu Youth Medical Talents Project (QNRC2016482) and the Six Talent Peak Projects in Jiangsu Province (WSW-304). These funding bodies were involved in the designing, collection, analysis and interpretation of data and in writing and publication of the manuscript. The authors have no other relevant affiliations or financial involvement with any organization or entity with a financial interest in or financial conflict with the subject matter or materials discussed in the manuscript apart from those disclosed.

No writing assistance was utilized in the production of this manuscript.

\section{Ethical conduct of research}

All animal experiments were carried out according to protocols approved by the Committee for Animal Research of Suqian Branch Jiangsu Province Hospital, China. The authors state that they have obtained appropriate institutional review board approval or have followed the principles outlined in the Declaration of Helsinki for animal experimental investigations.

\section{Open access}

This work is licensed under the Attribution-NonCommercial-NoDerivatives 4.0 Unported License. To view a copy of this license, visit http://creativecommons.org/licenses/by-nc-nd/4.0/

\section{References}

Papers of special note have been highlighted as: • of interest; $\bullet \bullet$ of considerable interest

1. Nagasaka M, Gadgeel SM. Role of chemotherapy and targeted therapy in early-stage non-small cell lung cancer. Expert Rev. Anticancer Ther. 18(1), 63-70 (2018).

2. Sakurada T. Analysis of risk factors for side effects and the establishment of supportive therapy during cancer chemotherapy. Yakugaku. Zasshi. 138(11), 1363-1370 (2018).

3. Eckhardt BL, Francis PA, Parker BS, Anderson RL. Strategies for the discovery and development of therapies for metastatic breast cancer. Nat. Rev. Drug Discov. 11(6), 479-497 (2012).

4. Chen S, Flower A, Ritchie A et al. Oral Chinese herbal medicine (CHM) as an adjuvant treatment during chemotherapy for non-small cell lung cancer: a systematic review. J. Eur. J. Integrat. Med. 68(2), 137-45 (2010).

-. Summarizes the traditional Chinese medicines used as adjuvant therapy for lung cancer treatment.

5. Liu R, He SL, Zhao YC et al. Chinese herbal decoction based on syndrome differentiation as maintenance therapy in patients with extensive-stage small-cell lung cancer: an exploratory and small prospective cohort study. Evid. Based Complement. Alternat. Med. 2015, 601-067 (2015).

6. Li SG, Chen HY, Ou-Yang CS et al. The efficacy of Chinese herbal medicine as an adjunctive therapy for advanced non-small cell lung cancer: a systematic review and meta-analysis. PLoS ONE 8(2), e57604 (2013).

7. Zhang XW, Liu W, Jiang HL, Mao B. Chinese herbal medicine for advanced non-small-cell lung cancer: a systematic review and meta-analysis. Am. J. Chin. Med. 46(5), 923-952 (2018).

8. Zhang JH, Zhu Y, Fan XH, Zhang BL. Efficacy-oriented compatibility for component-based Chinese medicine. Acta. Pharmacol. Sin. 36(6), 654-658 (2015).

9. Siman P, Filipova A, Ticha A, Niang M, Bezrouk A, Havelek R. Effective method of purification of betulin from birch bark: the importance of its purity for scientific and medicinal use. PLoS ONE 11(5), e0154933 (2016).

10. Simone F, Guido K. Targeting mitochondrial apoptosis by betulinic acid in human cancers. Drug Discov. Today 14(17-18), 885-890 (2009).

11. Pareek A, Suthar M, Rathore GS, Bansal V. Feverfew (Tanacetum parthenium L.): a systematic review. Pharmacogn. Rev. 5(9), 103-110 (2011).

12. Akram G, Ansam S, Zdenko H, Nadine D. Parthenolide: from plant shoots to cancer roots. Drug Discov. Today 18(17-18), 894-905 (2013).

13. Lo YC, Teng CM, Chen CF, Chen CC, Hong CY. Magnolol and honokiol isolated from magnolia officinalis protect rat heart mitochondria against lipid peroxidation. Biochem. Pharmacol. 47(3), 549-553 (1994).

14. Yeh PS, Wang W, Chang YA, Lin CJ, Wang JJ, Chen RM. Honokiol induces autophagy of neuroblastoma cells through activating the PI3K/Akt/mTOR and endoplasmic reticular stress/ERK1/2 signaling pathways and suppressing cell migration. Cancer Lett. 370(1), 66-77 (2016).

15. Smith I, Williamson EM, Putnam S, Farrimond J, Whalley BJ. Effects and mechanisms of ginseng and ginsenosides on cognition. Nutr. Rev. 72(5), 319-333 (2014).

16. Chen W, Qiu Y. Ginsenoside Rh2 targets EGFR by up-regulation of miR-491 to enhance anti-tumor activity in hepatitis B cvirus-related hepatocellular carcinoma. Cell. Biochem. Biophys. 72(2), 325-331 (2015). 
17. Lei M, Ma G, Sha S et al. Dual-functionalized liposome by co-delivery of paclitaxel with sorafenib for synergistic antitumor efficacy and reversion of multidrug resistance. Drug Deliv. 26(1), 262-272 (2019).

18. Gong Z, Chen D, Xie F et al. Codelivery of salinomycin and doxorubicin using nanoliposomes for targeting both liver cancer cells and cancer stem cells. Nanomedicine (Lond.) 11(19), 2565-2579 (2016).

19. Zhang C, Zhang S, Zhi D, Cui J. Cancer treatment with liposomes based drugs and genes co-delivery systems. Curr. Med. Chem. 25(28), 3319-3332 (2018).

20. Zununi Vahed S, Salehi R, Davaran S, Sharifi S. Liposome-based drug co-delivery systems in cancer cells. Mater. Sci. Eng. C Mater. Biol. Appl. 71, 1327-1341 (2017).

- Summarizes the advantage of liposome-based codelivery systems.

21. Xiao W, Zeng X, Lin H, Han K, Jia HZ, Zhang XZ. Dual stimuli-responsive multi-drug delivery system for the individually controlled release of anti-cancer drugs. Chem. Commun. 51(8), 1475-1478 (2015).

22. Desale JP, Swami R, Kushwah V, Katiyar SS, Jain S. Chemosensitizer and docetaxel-loaded albumin nanoparticle: overcoming drug resistance and improving therapeutic efficacy. Nanomedicine (Lond.) 13(21), 2759-2776 (2018).

23. AbdElhamid AS, Helmy MW, Ebrahim SM et al. Layer-by-layer gelatin/chondroitin quantum dots-based nanotheranostics: combined rapamycin/celecoxib delivery and cancer imaging. Nanomedicine (Lond.) 13(14), 1707-1730 (2018).

24. Jin X, Li M, Yin L, Zhou J, Zhang Z, Lv H. Tyroservatide-TPGS-paclitaxel liposomes: tyroservatide as a targeting ligand for improving breast cancer treatment. Nanomedicine 13(3), 1105-1115 (2017).

25. Chou TC. Theoretical basis, experimental design, and computerized simulation of synergism and antagonism in drug combination studies. Pharmacol. Rev. 58(3), 621-681 (2006).

- Summarizes the combination index calculated by using the Chou-Talalay method.

26. Selby LI, Cortez-Jugo CM, Such GK, Johnston APR. Nanoescapology: progress toward understanding the endosomal escape of polymeric nanoparticles. Wiley. Interdiscip. Rev. Nanomed. Nanobiotechnol. 9(5), e1452 (2017).

27. Zhang J, Zheng Y, Xie X et al. Cleavable multifunctional targeting mixed micelles with sequential $\mathrm{pH}$-triggered TAT peptide activation for improved antihepatocellular carcinoma efficacy. Mol. Pharm. 14(11), 3644-3659 (2017).

28. Zhang J, Li J, Shi Z et al. pH-Sensitive polymeric nanoparticles for co-delivery of doxorubicin and curcumin to treat cancer via enhanced pro-apoptotic and anti-angiogenic activities. Acta. Biomater. 58, 349-364 (2017).

29. Cai A, Wang CH, Yan MM et al. A liposome preparation based on $\beta$-CD-LPC molecule and its application as drug-delivery system. Nanomedicine (Lond.) 13(21), 2777-2789 (2018).

30. Rodriguez MA, Pytlik R, Kozak T et al. Vincristine sulfate liposomes injection (Marqibo) in heavily pretreated patients with refractory aggressive non-Hodgkin lymphoma: report of the pivotal Phase II study. Cancer 115(15), 3475-3482 (2009).

31. Barenholz Y. Doxil ${ }^{\circledR}$ - the first FDA-approved nano-drug: lessons learned. J. Control. Rel. 160(2), 117-134 (2012).

32. Coleman RE, Biganzoli L, Canney P et al. A randomised Phase II study of two different schedules of pegylated liposomal doxorubicin in metastatic breast cancer (EORTC-10993). Eur. J. Cancer 42(7), 882-887 (2016).

33. Mu LM, Ju RJ, Liu R et al. Dual-functional drug liposomes in treatment of resistant cancers. Adv. Drug Deliv. Rev. 115, 46-56 (2017).

34. Marucci C, Fumagalli G, Calogero F et al. Natural products and cancer stem cells. Curr. Pharm. Des. 21(38), 5547-5557 (2015).

35. Bonofiglio D, Giordano C, De Amicis F, Lanzino M, Andò S. Natural products as promising antitumoral agents in breast cancer: mechanisms of action and molecular targets. Mini. Rev. Med. Chem. 16(8), 596-604 (2016).

36. Catanzaro E, Greco G, Potenza L, Calcabrini C, Fimognari C. Natural products to fight cancer: a focus on Juglans regia. Toxins (Basel) 10(11), E469 (2018).

37. Cai Y, Zhang J, Chen NG et al. Recent advances in anticancer activities and drug delivery systems of tannins. Med. Res. Rev. 37(4), 665-701 (2017).

38. Vogelzang NJ, Benowitz SI, Adams S et al. Clinical cancer advances 2011: annual report on progress against cancer from the American Society of Clinical Oncology. J. Clin. Oncol. 30(1), 88-109 (2012).

39. Lee H, Lee S, Jeong D, Kim SJ. Ginsenoside Rh2 epigenetically regulates cell-mediated immune pathway to inhibit proliferation of MCF-7 breast cancer cells. J. Ginseng. Res. 42(4), 455-462 (2018).

40. Jafari N, Nazeri S, Enferadi ST. Parthenolide reduces metastasis by inhibition of vimentin expression and induces apoptosis by suppression elongation factor $\alpha-1$ expression. Phytomedicine 41, 67-73 (2018).

41. Kumar P, Bhadauria AS, Singh AK, Saha S. Betulinic acid as apoptosis activator: molecular mechanisms, mathematical modeling and chemical modifications. Life Sci. 209, 24-33 (2018).

42. Sun L, Cao J, Chen K et al. Betulinic acid inhibits stemness and EMT of pancreatic cancer cells via activation of AMPK signaling. Int. J. Oncol. 54(1), 98-110 (2019).

43. Chiu CS, Tsai CH, Hsieh MS et al. Exploiting honokiol-induced ER stress CHOP activation inhibits the growth and metastasis of melanoma by suppressing the MITF and $\beta$-catenin pathways. Cancer Lett. 442, 113-125 (2019). 
44. Carlisi D, De Blasio A, Drago-Ferrante R et al. Parthenolide prevents resistance of MDA-MB231 cells to doxorubicin and mitoxantrone: the role of Nrf2. Cell Death Discov. 3, 17078 (2017).

45. Liu GW, Liu YH, Jiang GS, Ren WD. The reversal effect of ginsenoside Rh2 on drug resistance in human colorectal carcinoma cells and its mechanism. Hum. Cell 31(3), 189-198 (2018).

46. Rauf A, Patel S, Imran M et al. Honokiol: an anticancer lignan. Biomed. Pharmacother. 107, 555-562 (2018).

47. Kohli AG, Kivimae S, Tiffany MR, Szoka FC. Improving the distribution of Doxil (R) in the tumor matrix by depletion of tumor hyaluronan. J. Control. Rel. 191, 105-114 (2014).

48. Herbert SP, Stainier DY. Molecular control of endothelial cell behaviour during blood vessel morphogenesis. Nat. Rev. Mol. Cell Biol. 12(9), 551-564 (2011).

49. Fang J, Nakamura H, Maeda H. The EPR effect: unique features of tumor blood vessels for drug delivery, factors involved, and limitations and augmentation of the effect. Adv. Drug Deliv. Rev. 63(3), 136-151 (2011).

50. Golombek SK, May JN, Theek B et al. Tumor targeting via EPR: strategies to enhance patient responses. Adv. Drug. Deliv. Rev. 130, 17-38 (2018).

51. Zhao C, Deng H, Xu J et al. "Sheddable" PEG-lipid to balance the contradiction of PEGylation between long circulation and poor uptake. Nanoscale 8(20), 10832-42 (2016).

\section{- Illustrates an example of PEGylation function.}

